\title{
Fabrication of Ti-Alloy Powder/Solid Composite with Uniaxial Anisotropy by Introducing Unidirectional Honeycomb Structure via Electron Beam Powder Bed Fusion
}

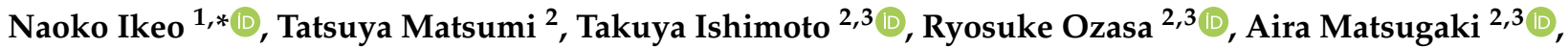 \\ Tadaaki Matsuzaka $^{2}$, Ozkan Gokcekaya ${ }^{2,3}$ (D), Yorinobu Takigawa ${ }^{4}$ and Takayoshi Nakano ${ }^{2,3, *(D)}$
}

1 Department of Mechanical Engineering, Graduate School of Engineering, Kobe University, 1-1 Rokkodai-cho, Kobe 657-8501, Hyogo, Japan

2 Division of Materials and Manufacturing Science, Graduate School of Engineering, Osaka University, 2-1 Yamadaoka, Suita 565-0871, Osaka, Japan; tatsuya.matsumi@mat.eng.osaka-u.ac.jp (T.M.); ishimoto@mat.eng.osaka-u.ac.jp (T.I.); ozasa@mat.eng.osaka-u.ac.jp (R.O.); matsugaki@mat.eng.osaka-u.ac.jp (A.M.); tadaaki.matsuzaka@mat.eng.osaka-u.ac.jp (T.M.); ozkan@mat.eng.osaka-u.ac.jp (O.G.)

3 Anisotropic Design \& Additive Manufacturing Research Center, Osaka University, 2-1 Yamadaoka, Suita 565-0871, Osaka, Japan

4 Department of Materials Science, Graduate School of Engineering, Osaka Prefecture University, 1-1 Gakuen-cho, Sakai 599-8531, Osaka, Japan; takigawa@mtr.osakafu-u.ac.jp

check for updates

Citation: Ikeo, N.; Matsumi, T.;

Ishimoto, T.; Ozasa, R.; Matsugaki, A.; Matsuzaka, T.; Gokcekaya, O.; Takigawa, Y.; Nakano, T. Fabrication of Ti-Alloy Powder/Solid Composite with Uniaxial Anisotropy by Introducing Unidirectional Honeycomb Structure via Electron Beam Powder Bed Fusion. Crystals 2021, 11, 1074. https://doi.org/ 10.3390/cryst11091074

Academic Editors: Umberto Prisco, Pan Wang and Jiaming Bai

Received: 21 August 2021

Accepted: 2 September 2021

Published: 5 September 2021

Publisher's Note: MDPI stays neutral with regard to jurisdictional claims in published maps and institutional affiliations.

Copyright: () 2021 by the authors. Licensee MDPI, Basel, Switzerland. This article is an open access article distributed under the terms and conditions of the Creative Commons Attribution (CC BY) license (https:// creativecommons.org/licenses/by/ $4.0 /)$.
* Correspondence: ikeo@mech.kobe-u.ac.jp (N.I.); nakano@mat.eng.osaka-u.ac.jp (T.N.); Tel.: +81-6-6879-7505 (N.I.); +81-78-803-6121 (T.N.)

Abstract: In this study, a Ti-6Al-4V alloy composite with uniaxial anisotropy and a hierarchical structure is fabricated using electron beam powder bed fusion, one of the additive manufacturing techniques that enable arbitrary fabrication, and subsequent heat treatment. The uniaxial anisotropic deformation behavior and mechanical properties such as Young's modulus are obtained by introducing a unidirectional honeycomb structure. The main feature of this structure is that the unmelted powder retained in the pores of the honeycomb structure. After appropriate heat treatment at $1020^{\circ} \mathrm{C}$, necks are formed between the powder particles and between the powder particles and the honeycomb wall, enabling a stress transmission through the necks when the composite is loaded. This means that the powder part has been mechanically functionalized by the neck formation. As a result, a plateau region appears in the stress-strain curve. The stress transfer among the powder particles leads to the cooperative deformation of the composites, contributing to the excellent energy absorption capacity. Therefore, it is expected that the composite can be applied to bone plates on uniaxially oriented microstructures such as long bones owing to its excellent energy absorption capacity and low elasticity to unidirectionally suppress stress shielding.

Keywords: Ti-6Al-4V; hierarchical composite; metal additive manufacturing; mechanical properties; energy absorption

\section{Introduction}

During the deformation of natural structural materials consisting of complex hierarchical structures, such as bones, almost all natural creations perform the necessary mechanical functions for the required orientation [1]. By mimicking the structure of natural creations, it would be beneficial to introduce structural hierarchy and functional anisotropy into metallic materials as well, in order to make them more functional. Therefore, in the field of biomaterials and aeronautical materials, adaptation to complex and anisotropic stress distributions is required. For example, bone tissue forms an anisotropic microstructure that supports the in vivo three-dimensional stress field generated by daily living [2,3]. As this difference in anisotropy and mechanical properties between bone and metal leads to a bone loss [4] and degradation of bone quality of the surrounding bone [5], it is important 
to develop materials with bone-like anisotropy and mechanical properties for the development of superior medical devices [6-8]. In recent years, mechanical properties have been controlled by hierarchical structures using additive manufacturing, which is good at designing arbitrary shapes [9-16], and material surface treatment [17-21].

Our previous research achieved a Ti-based material with a hierarchical structure by powder/solid compositing using electron-beam powder bed fusion (EB-PBF) [11]. The introduction of the powder structure into a void of a porous structure exhibiting a low Young's modulus, and the stress transmission between both structures by neck formation, contribute to both the low Young's modulus and the excellent energy absorption capability. By further providing uniaxial anisotropy to the structure and arranging it in 3-D, it is expected that the structure can freely exhibit mechanical functions in various directions in a living body, where complicated stress distribution occurs. However, in the past powder/solid composites, a unidirectional pore was introduced in the solid structure, based on previous research on bone regeneration with unidirectional scaffolds [22-24], and its anisotropy has not yet been sufficiently evaluated.

In the case of in-plane compression of a unit cell with square prisms, as in our previous report, it is known that the mechanical properties are strongly dependent on the compression direction. In contrast, in the in-plane deformation of a structure with regular hexagonal columnar pores or honeycomb pores, the mechanical properties are isotropic [25]. Therefore, the preparation of a powder/solid composite structure with unidirectional honeycomb pores can be expected to produce structures exhibiting uniaxial anisotropy, low Young's modulus, and high energy absorption capacity.

In this study, open-porous structures containing unidirectional honeycomb pores and powder/solid composite structures were fabricated using the EB-PBF technique. This study also attempted to elucidate the contribution of the introduction of unidirectional honeycomb pores to the deformation mechanism and anisotropy of mechanical properties, as well as the contribution of powder/solid composite structures to the deformation mechanism and uniaxial anisotropy.

\section{Materials and Methods}

The three-dimensional model of the honeycomb structure shown in Figure 1 was prepared using computer-aided design (CAD) software (SolidWorks, Dassault Systèmes SolidWorks Corp., Waltham, MA, USA). The external shape of each structure was $10 \times 10 \times 15 \mathrm{~mm}^{3}$. The four honeycomb pores could be arranged parallel to one side. The CAD model (Figure 1a) in which the honeycomb pores penetrate in the longitudinal direction was used to manufacture the honeycomb porous structure. The CAD model (Figure 1c) in which a thin plate was applied to the bottom to maintain the unmelted residual powder particles in the honeycomb pores was used to manufacture the honeycomb composite structure. Hereinafter, the structure produced based on the CAD model shown in Figure 1a is referred to as a porous specimen, and the structure produced based on the CAD model shown in Figure $1 \mathrm{c}$ is referred to as a composite specimen.

Based on these data, a three-dimensional structure was fabricated using an EB-PBF apparatus (Arcam Q10, ArcamAB, Mölndal, Sweden). To build the specimen, the same Ti6Al-4V ELI alloy powder was used. The building direction was parallel to the honeycomb pore extension direction. The beam voltage, beam current, scan interval, layer thickness, and scan speed were $60 \mathrm{kV}, 1.5 \mathrm{~mA}, 0.1 \mathrm{~mm}, 70 \mu \mathrm{m}$, and $100 \mathrm{~mm} / \mathrm{s}$, respectively. The porosity and powder packing ratio were calculated from the size and mass of the specimens. A theoretical density of $4.43 \mathrm{~g} / \mathrm{cm}^{3}$ of Ti-6Al-4V ELI was used to derive both parameters.

The prepared specimens were heat-treated in an argon gas atmosphere using a boxtype electric furnace (MBS 13-2040, Motoyama, Osaka, Japan). The heat treatment was performed at $1020^{\circ} \mathrm{C}$ for $100 \mathrm{~h}$ to sufficiently promote neck formation between powder particles. After heat treatment, the samples were quenched in ice water. 
(a)

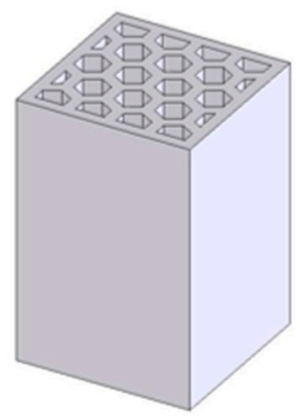

(c)

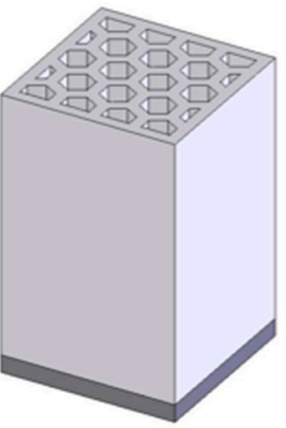

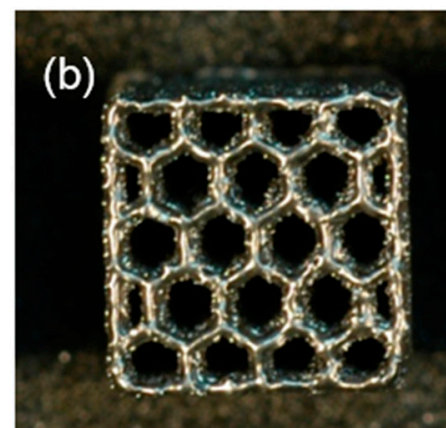

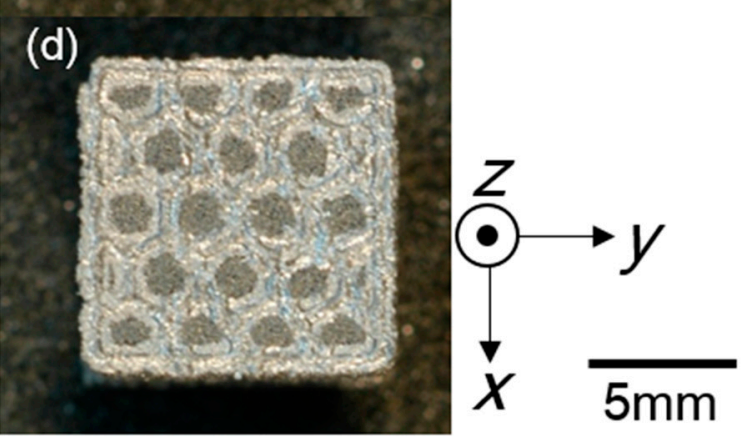

Figure 1. Three dimensional image of CAD model and pictures of specimen top surface for $(\mathbf{a}, \mathbf{b})$ porous specimen with elongated honeycomb pores and (c,d) powder/solid composite with un-melted powder particles within the pores (composite specimen).

To eliminate the difference in dimensions with respect to the compression axis described later, the sample was cut off at the top and bottom, and made into a cube of $10 \mathrm{~mm}$ each side. The non-destructive observation of the internal structure of the fabricated specimen, was conducted using a micro-focus X-ray CT apparatus (SMX-100CT, Shimadzu, Kyoto, Japan). The measurement conditions, namely the tube voltage, tube current, and resolution, were $100 \mathrm{kV}, 25 \mathrm{~mA}$, and $34.7 \mu \mathrm{m}$, respectively.

The constituent phases were identified using a wide-area X-ray diffractometer ( $X^{\prime}$ Pert PRO, PANalytical, Almelo, The Netherlands). The $\mathrm{X}$-ray source was $\mathrm{Cu}-\mathrm{K} \alpha$ radiation, and $X$-ray diffraction $(\mathrm{XRD})$ patterns were analyzed to determine the constituent phases. After electropolishing, the microstructure was observed using an optical microscope (DP-51, Olympus, Tokyo, Japan). In addition, scanning electron microscopy (SEM) (JSM-5600, JEOL, Tokyo, Japan) was used to observe the neck formation between the powder particles.

To evaluate the mechanical properties of these specimens, a quasi-static uniaxial compression test was conducted using a compression testing machine (AG- $X$, Shimadzu, Kyoto, Japan). Compression tests were performed in two directions: parallel and perpendicular to the pore extension direction $(N=3)$. A dial gauge was used to measure the strain during deformation, and the initial strain rate was $1.67 \times 10^{-4} \mathrm{~s}^{-1}$. A contact strain gauge was used to precisely measure the Young's modulus. The plateau stress was determined as the average stress value between 20 and 30\% strain according to JIS H 7902:2016. Equation (1) was used to calculate the toughness $(W)$ as an index of energy absorption, where $e$ is the nominal strain, $s$ is the nominal stress, and $e_{\mathrm{d}}$ is the nominal strain at the densification of the samples. The method of deriving the densification strain was previously described [11].

$$
W=\int_{0}^{e_{\mathrm{d}}} s(e) d e
$$

\section{Results}

\subsection{Densities and Microstructures of Specimens}

Figure $1 b$,d shows the appearance of the porous and composite specimens fabricated using EB-PBF. Figure 2 shows $\mu$-CT images of both specimens. The extended honeycomb pores in the building direction (Z-axis) is clearly seen in both the porous and composite 
specimens. As shown in Figure 2c,d, the unmelted powder is present inside the pores of the composite specimen, which can be determined from the fact that the gray value of the CT image is between that of pore and solid part. The relative densities of the porous and composite specimens were $49.4 \pm 0.6 \%$ and $66.7 \pm 0.9 \%$, respectively, using the density of the Ti-6Al-4V alloy $\left(4.43 \mathrm{~g} / \mathrm{cm}^{3}\right)$. From these densities, the packing density of the powder inside the pores was calculated to be $42.0 \pm 1.8 \%$.
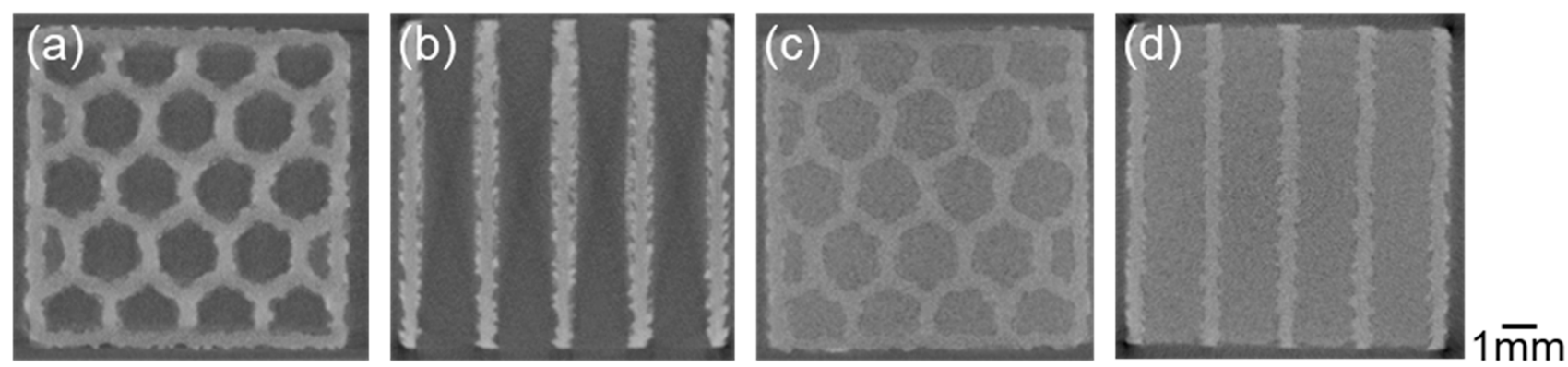

Figure 2. $\mu$-CT cross-section images for $(\mathbf{a}, \mathbf{b})$ porous specimen with elongated honeycomb pores and (c,d) powder/solid composite specimen with residual unmelted powder particles inside the pores.

Figure 3 shows the XRD pattern and an optical micrograph of the specimen after heat treatment. The XRD pattern (Figure 3a) indicates that the specimens consist of $\alpha$ $\left(\right.$ or $\left.\alpha^{\prime}\right)+\beta$ phases. An acicular $\alpha / \alpha^{\prime}$ is observed in the optical micrograph taken in the plane parallel to the building direction (Figure $3 b$ ). Since the heat treatment was carried out at the temperature above the $\beta$ to $\alpha$ transformation point of $900-950{ }^{\circ} \mathrm{C}[26,27]$, the traces of primary $\beta$-grains elongated along the building direction (Z-axis), which have been frequently observed in PBF-fabricated specimens [28-30], disappeared.

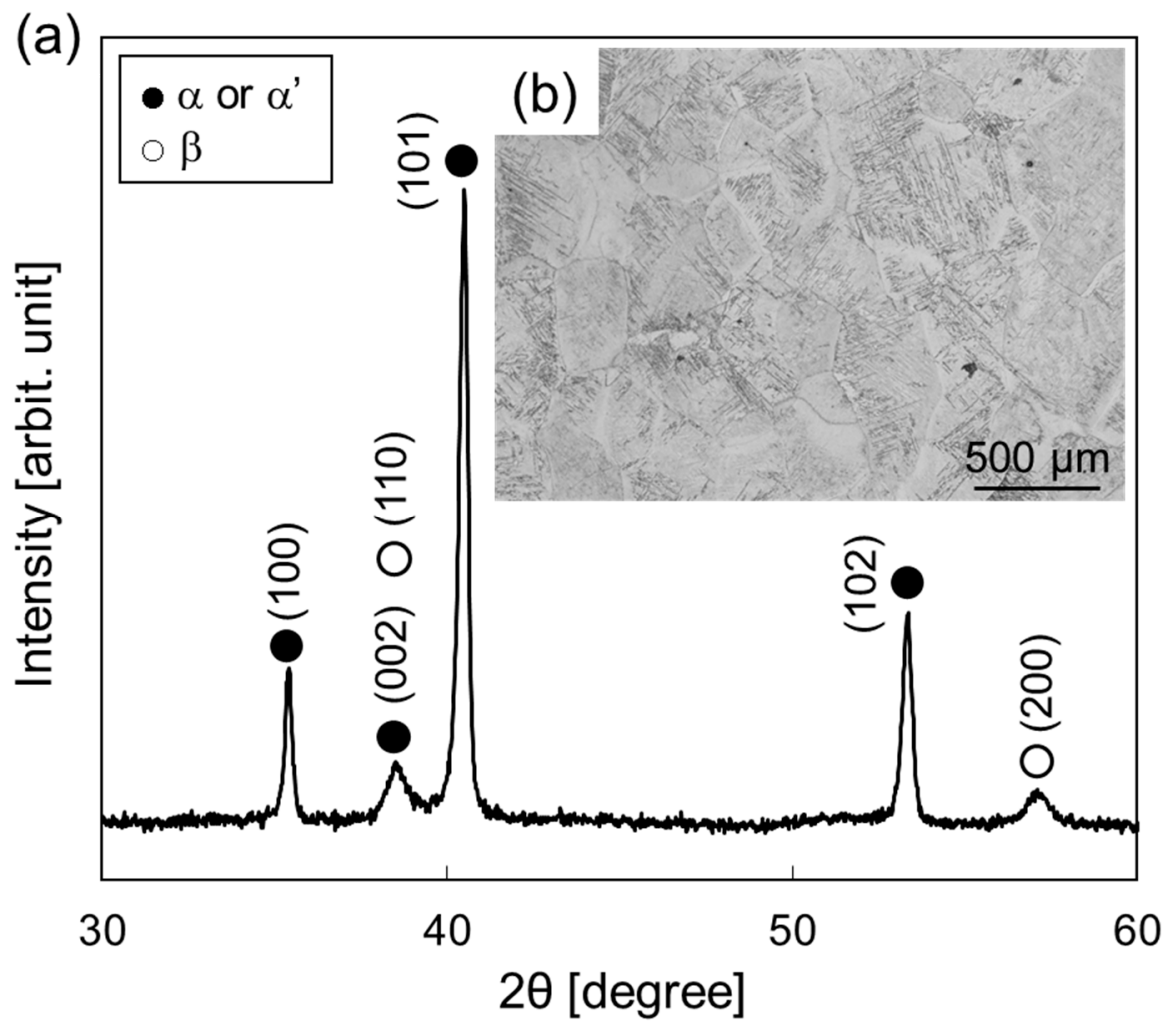

Figure 3. (a) XRD pattern and (b) optical micrograph of longitudinal sections of the fabricated specimen after heat treatment. In (b), the vertical direction corresponds to the building direction. 
Heat treatment resulted in the formation of necks between the powder particles (Figure 4), and powder particles construct a three-dimensional network through the necks. Necks also formed between the solid part and the powder particles, resulting in a hierarchical structure consisting of macroscopic solid wall and powder parts, which are structurally connected to each other. This allows the powder part to exert mechanical functions by transferring stress through the neck.

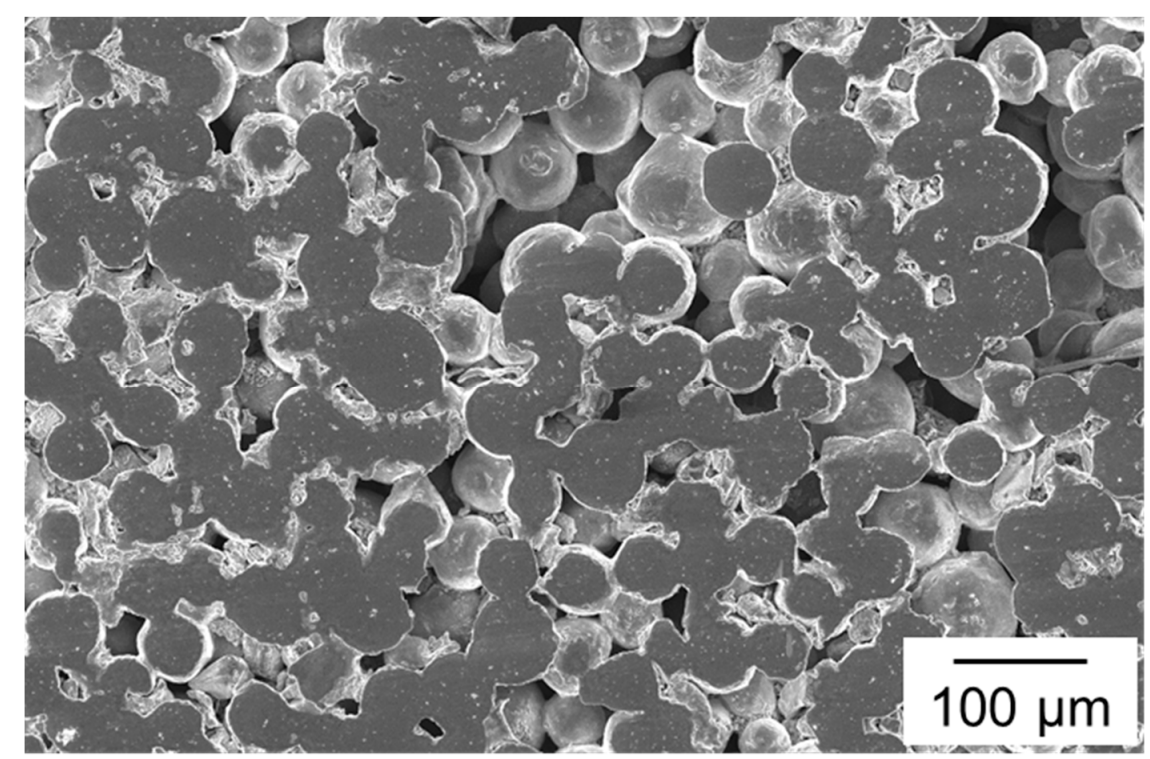

Figure 4. SEM micrograph of the powder part after heat treatment. A three-dimensional network is constructed through the necks between the powder particles.

\subsection{Deformation Behaviors and Mechanical Properties of the Products}

Figure 5a shows the stress-strain curves obtained by a uniaxial compression test of the porous specimen in the $Y$ - and Z-directions. A significant anisotropy in deformation behavior is observed. Young's modulus and $0.2 \%$ proof stress are significantly higher for compression in the Z-directions. When the porous specimen is compressed in the $Y$ direction perpendicular to the elongated pore, a characteristic oscillation in stress appears. Finally, the stress shows a rapid increase.

(a)

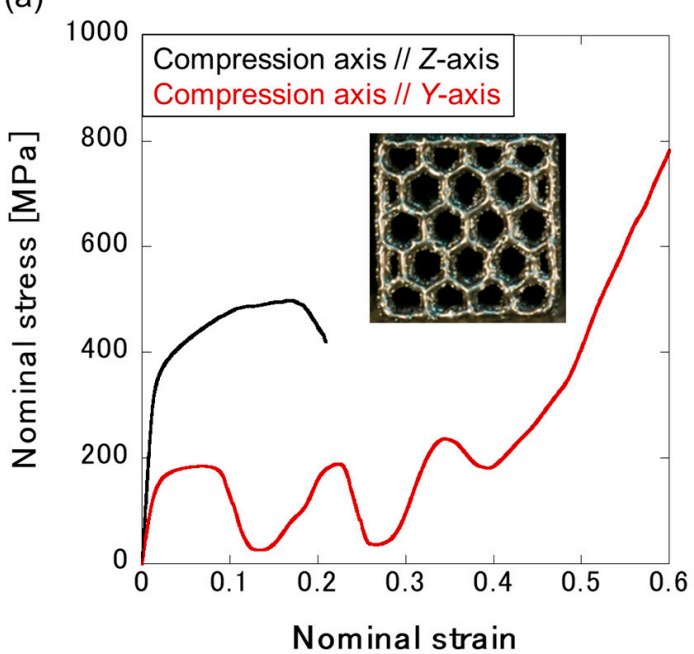

(b)

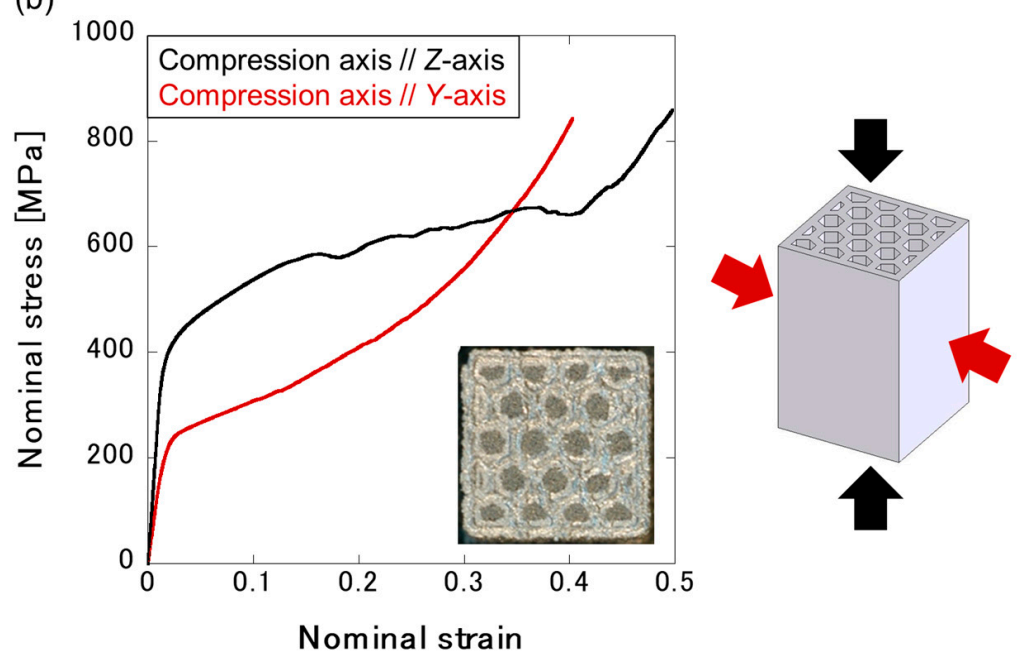

Figure 5. Orientation dependence of nominal stress-nominal strain curves in the Z-and $Y$-axis directions in compression at ambient temperature for (a) porous specimen and (b) composite specimen. 
As shown in Figure 5b, the powder/solid composite specimen shows different deformation behavior from the porous specimen. The anisotropy in Young's modulus and proof stress is similar to that of the porous specimen, however, the post yield deformation behavior is largely different from that of the porous specimen. Under the compression along Z-direction, the specimen does not reach a clear rupture and a plateau region of stress appears. When the composite specimen is compressed in the $Y$-direction, the stress oscillation seen in the porous specimen disappears and the stress increases continuously through a plateau-like region. Therefore, the heat-treated powder/solid composite acquired energy absorption capacity and fracture was avoided.

The mechanical properties obtained are listed in Table 1. Comparing the porous and composite specimens, the powder/solid structuring made moderate contribution to the Young's modulus and proof stress, which resulted in somewhat isotropic properties, but greatly improved the toughness (energy absorption capacity).

Table 1. Mechanical properties of porous and composite specimens fabricated by EB-PBF and subsequent heat treatment.

\begin{tabular}{ccccc}
\hline \multirow{2}{*}{ Compressive Axis } & \multicolumn{2}{c}{ Porous Specimen } & \multicolumn{2}{c}{ Composite Specimen } \\
& Z-Direction & Y-Direction & Z-Direction & Y-Direction \\
\hline Young's modulus [GPa] & $41.9 \pm 9.3$ & $28.9 \pm 1.1$ & $50.5 \pm 3.9$ & $38.1 \pm 2.9$ \\
0.2\% Proof stress [MPa] & $326 \pm 8$ & $120 \pm 13$ & $368 \pm 13$ & $210 \pm 20$ \\
Plateau stress [MPa] & - & - & $629 \pm 12$ & $455 \pm 26$ \\
Densification strain & - & - & $0.40 \pm 0.02$ & $0.29 \pm 0.00$ \\
Toughness [MPa] & $85.8 \pm 6.2$ & $16.0 \pm 1.8$ & $231 \pm 8$ & $101 \pm 12$ \\
Specific energy absorption [J/g] & $19.4 \pm 1.4$ & $3.6 \pm 0.4$ & $52.1 \pm 1.8$ & $22.8 \pm 0.1$ \\
\hline
\end{tabular}

\section{Discussion}

4.1. Effect of Powder/Solid Structuring on the Anisotropy Deformation Behavior of Porous Specimens with Unidirectional Honeycomb Pores

The deformation behavior of the porous specimen exhibited a pronounced dependence on the loading direction. When the specimen was compressed in the Z-direction, cracks formed on the diagonal of the wall along the maximum shear stress direction (data not shown). The porous specimens fractured after reaching the ultimate stress, with buckling of the internal solid wall.

In contrast, when the porous specimen was compressed in the $Y$-direction, local plastic deformation was observed. At the first stress drop, the wall locally deformed, and the aligned pores crushed along the direction of the maximum shear stress, which existed on the diagonal of the $X Y$-plane (Figure 6b). Upon further strain, the adjacent layer of aligned pores crushed (Figure 6c,d). Eventually, the pores crushed completely, i.e., densification was achieved.

The period (strain) of the stress-strain curve predicted from pore diameter was $0.17 \pm 0.01 \%$. The measured period from the stress-strain curve (Figure 5a) is $0.15 \pm 0.01$, which well agrees with the estimated value. Therefore, the peculiar deformation behavior of the porous specimen when compressed in the $Y$-axis was considered to be caused by the crushing of the local pores as the deformation progressed. The contribution of partial pore crushing to such deformation behavior has been reported in $\mathrm{Al}$ foam and various honeycomb structures made by additive manufacturing [31-34].

The stress-strain curve of the porous honeycomb structure was completely changed by the powder/solid structuring. In the deformation of the composite specimen along the $Y$-axis, the stress did not show any decrease after yielding, but increased smoothly with the progress of deformation until final densification (Figure $5 b$ ). The wavy stress observed in the porous specimen was not observed. The local pore crush observed in the $Y$-axis compression of the porous specimen did not occur during deformation for the composite specimen. In the late stage of deformation, multiple shear zones were formed obliquely (Figure $6 \mathrm{~g}, \mathrm{~h}$ ), similar to the deformation of the porous specimens. In the powder/solid structure, the presence of necking between the powder particles and between the powder 
and solid parts contributed to the stress transfer. This indicates that the neck formation mechanically functionalized the powder part. To quantitatively evaluate the effect of the powder/solid structure on the reduction of deformation periodicity, stress distribution analysis during elastic deformation was conducted using the finite element method (FEM).

(a)

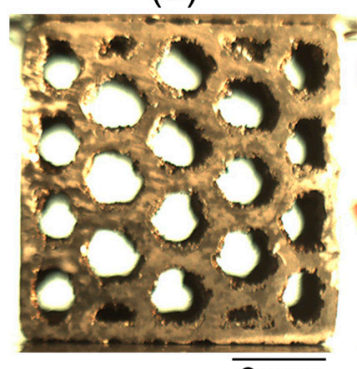

(e)

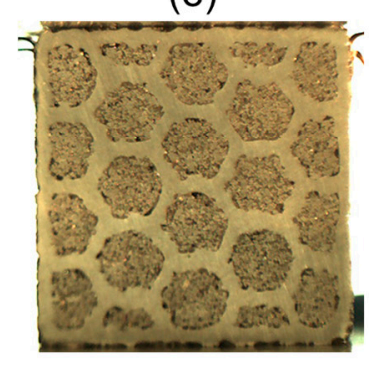

Initial (b)

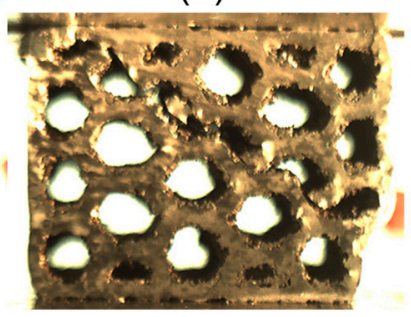

(f)

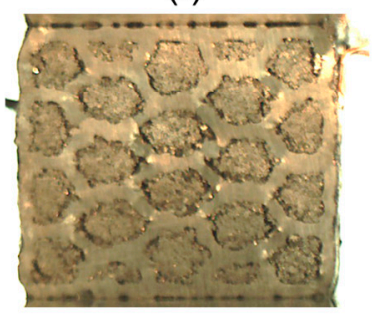

Strain 0.15 (c)

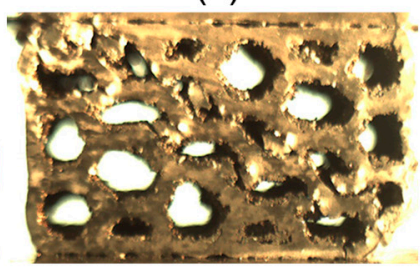

(d)

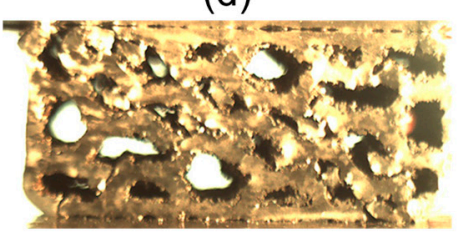

(g)

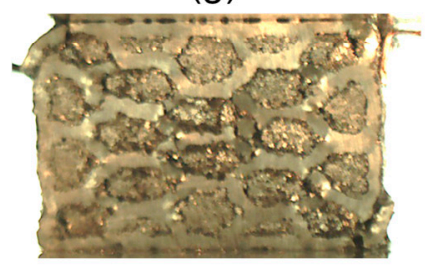

Strain 0.27 (h)

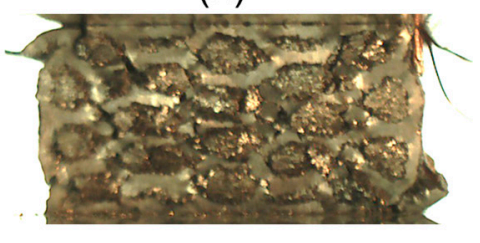

Strain 0.40

Figure 6. Compressive deformation behavior of (a-d) porous and (e-h) composite specimen.

The CAD data shown in Figure $1 \mathrm{a}, \mathrm{b}$ were used as an FEM model. The network structure of the powder particles was not reproduced in the model of the composite structure. A material with low Young's modulus was assumed as the neck-formed powder part. The analysis software used was Femap with Nastran (Siemens, Munich, Germany) and a hexagonal mesh was used. The porous model had 464,572 nodes and 96,987 elements, whereas the composite model had 1,301,480 nodes and 244,406 elements. The bottom edge of the model was fully constrained, and the top edge was loaded. The mechanical properties of the solid walls of the honeycomb structure were set to a Young's modulus of 110 GPa and a Poisson's ratio of 0.3. The Young's modulus of the neck-formed powder part $\left(E_{\text {Powder }}\right)$ was estimated by a rule of mixture in a parallel geometry, $E_{\text {Composite }}=$ $A_{\text {Solid }} E_{\text {Solid }}+A_{\text {Powder }} E_{\text {Powder }}=E_{\text {Porous }}+\left(1-A_{\text {Solid }}\right) E_{\text {Powder }}$, using the Young's moduli of porous $\left(E_{\text {Porous }}\right)$ and composite $\left(E_{\text {Composite }}\right)$ specimens, and the area fraction of solid wall $\left(A_{\text {Solid }}\right)$ and powder parts $\left(1-A_{\text {Solid }}\right)$ when compressed in the $Z$ direction. The estimated modulus of the neck-formed powder part was $18.7 \mathrm{GPa}$, therefore, $20 \mathrm{GPa}$ was used in the simulation.

The resulting von Mises stress distribution is shown in Figure 7. It is apparent that the stress distribution changes significantly, depending on the presence or absence of the networked powder structure. Although this analysis is about elastic deformation, the porous structure causes a local stress concentration at the inner side of the bend of the honeycomb walls (Figure 7a). In actual deformation, a shear zone passing through the stress concentration point may be formed, resulting in periodic deformation. However, in the composite structure, the stress concentration is relieved (Figure $7 \mathrm{~b}$ ) owing to the stress transfer between the powder and solid parts. Therefore, it is considered that the formation of the powder/solid structure resulted in a coordinated deformation of the entire structure and changed the deformation behavior. In other words, it can be inferred that the introduced powder structure not only exerts higher deformation stress than the porous 
structure, but also contributes to the loss of stress fluctuation by exerting stress transfer capability.

(a)

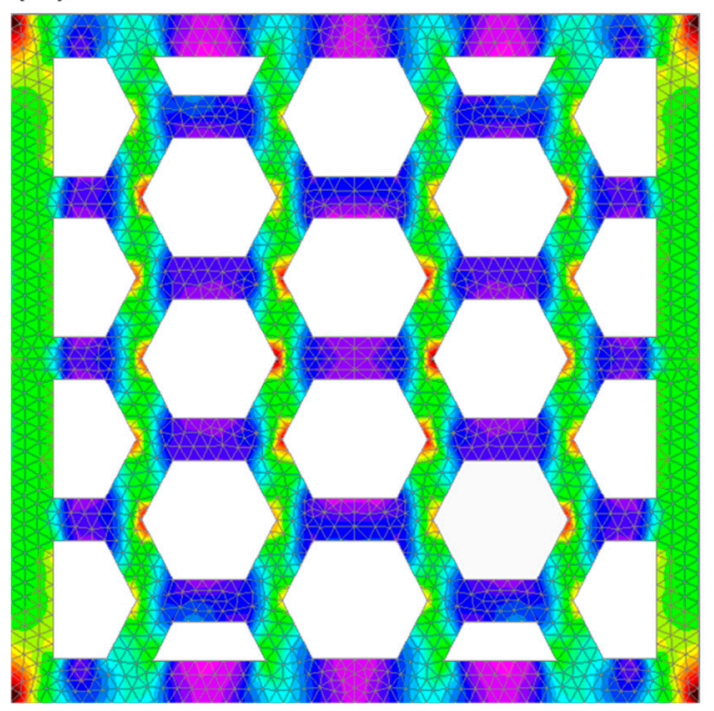

(b)

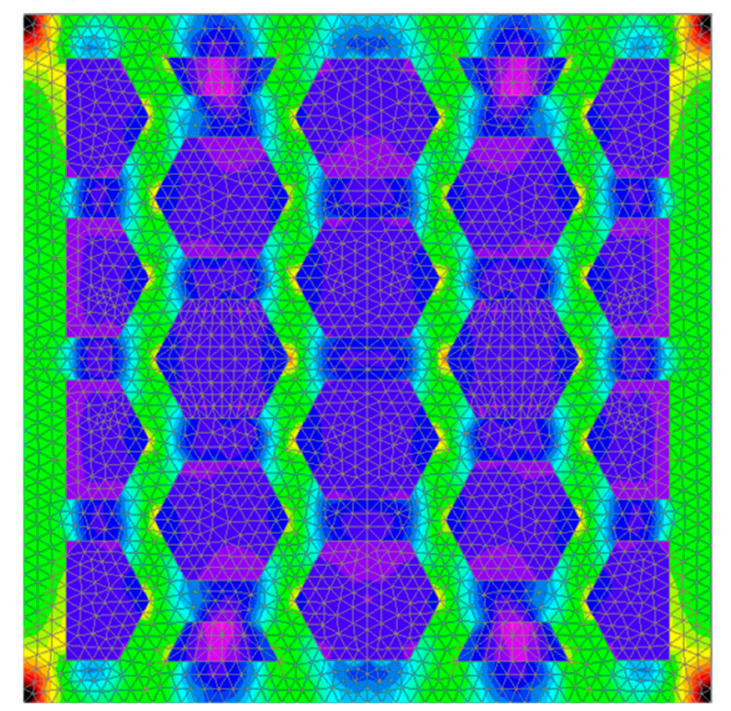

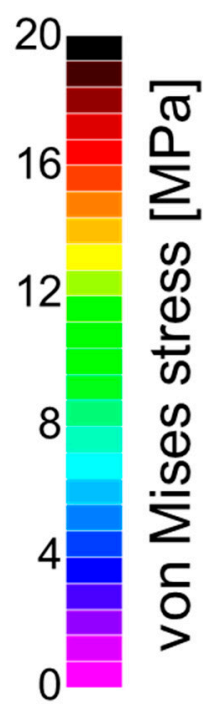

Figure 7. von Misses stress distribution for (a) porous and (b) composite structure.

\subsection{Influence of Honeycomb Pore Introduction and Powder/Solid Structuring on Anisotropy of Mechanical Properties}

In general, the Young's modulus and yield stress of the porous structure are expressed by Equation (2):

$$
X=X_{0}(1-\rho)^{k}
$$

where $X$ is the mechanical property of the porous structure, $X_{0}$ is the mechanical property of the bulk material, $\rho$ is the porosity, and $k$ is the stress concentration factor, which varies with the pore shape. The ideal relationship between the mechanical properties (Z-axis) of the honeycomb structure and porosity is expressed as Equation (3) because $k=1$ when no stress concentration occurs at the wall when a load is applied. The Young's modulus and proof stress vary linearly with the effective cross-sectional area, i.e., the porosity.

$$
X=X_{0}(1-\rho)
$$

The theoretical values of Young's modulus and proof stress of the porous structure derived from Equation (3) were $55.7 \mathrm{GPa}$ and $404 \mathrm{MPa}$, respectively. These values were higher than the experimental values, probably because of the stress concentration on the porous specimen fabricated by EB-PBF. The cross-sectional image in Figure $2 b$ shows that the surface roughness of the wall part is large, and micro voids are formed in the solid wall. It is considered that the micrometer-order defects described above caused stress concentration in the load-bearing solid wall, resulting in a decreased Young's modulus and proof stress of the porous specimen compared to the theoretical values.

In the specimen fabricated in this study, it is difficult to derive the mechanical properties by applying theoretical equations because the number of honeycomb pores is small, and partially incomplete honeycomb pores are introduced by the solid wall structure. Therefore, the Young's modulus was calculated using the FEM results shown earlier. The Young's modulus, when compressed in the $X$ - and $Y$-directions was $35.3 \mathrm{GPa}$ and $32.9 \mathrm{GPa}$, respectively. These values are slightly higher than the experimental values. This may be because of the effect of the microdefects mentioned earlier. However, the values were close to the experimental values, although there were slight differences. Therefore, it was confirmed that the Young's modulus could be controlled by the shape design of the unidirectional hon- 
eycomb pore. In addition, the FEM results show that the Young's modulus is approximately $30 \mathrm{GPa}$ in both the $X$ - and $Y$-axes. This indicates that by introducing a porosity of more than $50 \%$, a low Young's modulus equivalent to that of the bone (10-30 GPa) [3] could be obtained. It can be concluded that the uniaxial anisotropy of the mechanical properties of this structure can be imparted by the introduction of regular hexagonal honeycomb pores.

Because powders are isotropically packed and the bearing capacity of necked powder particles depends on the size of the neck [33], the stress in the powder part of the honeycomb pores is independent of the compressive direction. Therefore, the uniaxial anisotropy of the plateau stress depends on the anisotropy of the stress required to deform the solid wall. As mentioned above, even in a composite specimen containing necked powder particles, the proof and plateau stresses in Z-direction are higher than those in Y-direction.

Although porous materials with unidirectional honeycomb pores have attracted attention as excellent materials for shock absorption, they do not exhibit their energy absorption capabilities in compression parallel to the pore-elongated direction. Therefore, periodic stress fluctuations with increasing strain are observed [35], as also represented in this study. Nevertheless, the honeycomb pore containing the powder/solid composite specimen fabricated in this study showed improved energy absorption capacity, as the deformation proceeded without an abrupt drop in the stress, even in the $Y$-axis deformation. In fact, the formation of the powder/solid structure improved the toughness by approximately 2.7-fold and 6.3-fold under the compression in Z- and Y-directions, respectively, and the suppression of stress concentration by the powder/solid structure formation is considered to have led to a significant improvement in toughness. Such an improvement in toughness by infilling the pores with secondary phases has been reported, for example, in the case of resin interpenetration into metallic lattice [36]. One of the originality of this work is that we fabricated a composite consisting of two phases from the same starting material. Since the neck-formed powder part can be obtained by an irradiation of a beam with low energy [37], it is possible to obtain an energy absorptive composite in one process of EB-PBF.

As a result, the formation of the honeycomb pore structure accompanied by the interconnected powder network, using EB-PPF and subsequent heat treatment, were shown to be very effective for the arbitrary control of mechanical properties with uniaxial anisotropy. In addition, it is expected that the three-dimensional arrangement of the honeycomb pore structures fabricated in this study will facilitate the design of medical materials that are required to respond to in vivo stress distribution. As PBF is a bottom-up approach using powder as raw material, it allows for the creation of flexible shapes, and custom-made implants that conform the shape to the patient's skeleton have recently become an important topic [38,39]. The ability to control not only the external shape, but also the internal structure and the resulting mechanical properties will allow for customization regarding both external shape and function using PBF technology.

\section{Conclusions}

In this study, we designed a novel powder/solid composite with uniaxially anisotropic and hierarchical structure via EB-PBF and subsequent heat treatment. The unique feature of this composite is that the unmelted powder, which is normally removed, was retained inside the honeycomb pore and the powder was used to add new functionalities to the specimen. Heat treatment was carried out to connect the powder particles by neck formation to allow stress transfer between powder particles to functionalize the powder part as a load-bearing component. As a result, the composite with the necked powder part after heat treatment showed excellent energy absorption capacity, whereas the specimen before heat treatment did not. The use of powders in PBF can be a new strategy for imparting mechanical functionality to products.

Author Contributions: Conceptualization, T.N.; funding acquisition, T.N.; investigation, N.I., T.M. (Tatsuya Matsumi), T.I., R.O., A.M., T.M. (Tadaaki Matsuzaka) and O.G.; methodology, N.I., T.M. (Tatsuya Matsumi), R.O., A.M., T.M. (Tadaaki Matsuzaka) and O.G.; project administration, T.N.; software, N.I.; supervision, T.I., Y.T. and T.N.; validation, N.I., T.I. and T.N.; visualization, N.I.; 
writing—original draft, N.I.; writing—review and editing, T.I., Y.T. and T.N. All authors have read and agreed to the published version of the manuscript.

Funding: This study was supported by Grants-in-Aid for Scientific Research (JP18H05254 and 20K05132). This work was also partly supported by the Cross-Ministerial Strategic Innovation Promotion Program (SIP), Materials Integration for Revolutionary Design System of Structural Materials, Domain C1: "Development of Additive Manufacturing Process for Ni-based Alloy" from the Japan Science and Technology Agency (JST). The author (N.I.; T.I.; and T.N.) also appreciates financial support in part by the Light Metal Educational Foundation, Japan.

Data Availability Statement: The data that support the findings of this study are available from the corresponding author upon reasonable request.

Conflicts of Interest: The authors declare no conflict of interest.

\section{References}

1. Weiner, S.; Traub, W. Bone structure from ångstroms to microns. FASEB J. 1992, 6, 879-885. [CrossRef]

2. Nakano, T.; Kaibara, K.; Tabata, Y.; Nagata, N.; Enomoto, S.; Marukawa, E.; Umakoshi, Y. Unique alignment and texture of biological apatite crystallites in typical calcified tissues analyzed by micro-beam X-ray diffractometer system. Bone 2002, 31, 479-487. [CrossRef]

3. Ishimoto, T.; Kawahara, K.; Matsugaki, A.; Kamioka, H.; Nakano, T. Quantitative evaluation of osteocyte morphology and bone anisotropic extracellular matrix in rat femur. Calcif. Tissue Int. 2021. [CrossRef]

4. Huiskes, R.; Weinans, H.; van Rietbergen, B. The relationship between stress shielding and bone resorption around total hip stems and the effects of flexible materials. Clin. Orthop. Relat. Res. 1992, 274, 124-134. [CrossRef]

5. Noyama, Y.; Miura, T.; Ishimoto, T.; Itaya, T.; Niinomi, M.; Nakano, T. Bone loss and reduced bone quality of the human femur after total hip arthroplasty under stress-shielding effects by titanium-based implant. Mater. Trans. 2012, 53, 565-570. [CrossRef]

6. Kang, J.; Dong, E.; Li, D.; Dong, S.; Zhang, C.; Wang, L. Anisotropy characteristics of microstructures for bone substitutes and porous implants with application of additive manufacturing in orthopaedic. Mater. Des. 2020, 191, 108608. [CrossRef]

7. Ishimoto, T.; Hagihara, K.; Hisamoto, K.; Sun, S.-H.; Nakano, T. Crystallographic texture control of beta-type Ti-15Mo-5Zr-3Al alloy by selective laser melting for the development of novel implants with a biocompatible low Young's modulus. Scr. Mater. 2017, 132, 34-38. [CrossRef]

8. Ikeo, N.; Fukuda, H.; Matsugaki, A.; Inoue, T.; Serizawa, A.; Matsuzaka, T.; Ishimoto, T.; Ozasa, R.; Gokcekaya, O.; Nakano, T. 3D puzzle in cube pattern for anisotropic/isotropic mechanical control of structure fabricated by metal additive manufacturing. Crystals 2021, 11, 959. [CrossRef]

9. Nickels, L. AM and aerospace: An ideal combination. Met. Powder Rep. 2015, 70, 300-303. [CrossRef]

10. Tan, C.; Zou, J.; Li, S.; Jamshidi, P.; Abena, A.; Forsey, A.; Moat, R.J.; Essa, K.; Wang, M.; Zhou, K.; et al. Additive manufacturing of bio-inspired multi-scale hierarchically strengthened lattice structures. Int. J. Mach. Tools Manuf. 2021, 167, 103764. [CrossRef]

11. Ikeo, N.; Ishimoto, T.; Nakano, T. Novel powder/solid composites possessing low Young's modulus and tunable energy absorption capacity, fabricated by electron beam melting, for biomedical applications. J. Alloys Compd. 2015, 639, 336-340. [CrossRef]

12. Soro, N.; Saintier, N.; Merzeau, J.; Veidt, M.; Dargusch, M.S. Quasi-static and fatigue properties of graded Ti-6Al-4V lattices produced by Laser Powder Bed Fusion (LPBF). Addit. Manuf. 2021, 37, 101653.

13. Wang, P.; Li, X.; Jiang, Y.; Ling, M.; Nai, S.; Ding, J.; Wei, J. Electron beam melted heterogeneously porous micro lattices for metallic bone applications: Design and investigations of boundary and edge effects. Addit. Manuf. 2020, 36, 101566.

14. Yuan, L.; Ding, S.; Wen, C. Additive manufacturing technology for porous metal implant applications and triple minimal surface structures: A review. Bioact. Mater. 2019, 4, 56-70. [CrossRef]

15. Bobbert, F.S.L.; Lietaert, K.; Eftekhari, A.A.; Pouran, B.; Ahmadi, S.M.; Weinans, H.; Zadpoor, A.A. Additively manufactured metallic porous biomaterials based on minimal surfaces: A unique combination of topological, mechanical, and mass transport properties. Acta Biomater. 2017, 53, 572-584. [CrossRef] [PubMed]

16. Wang, P.; Li, X.; Luo, S.; Ling, M.; Nai, S.; Ding, J.; Wei, J. Additively manufactured heterogeneously porous metallic bone with biostructural functions and bone-like mechanical properties. J. Mater. Sci. Technol. 2021, 62, 173-179. [CrossRef]

17. Sugino, A.; Ohtsuki, C.; Tsuru, K.; Hayakawa, S.; Nakano, T.; Okazaki, Y.; Osaka, A. Effect of spatial design and thermal oxidation on apatite formation on Ti-15Zr-4Ta-4Nb alloy. Acta Biomater. 2008, 5, 298-304. [CrossRef]

18. Matsugaki, A.; Aramoto, G.; Nakano, T. The alignment of MC3T3-E1 osteoblasts on steps of slip traces introduced by dislocation motion. Biomaterials 2012, 33, 7327-7335. [CrossRef] [PubMed]

19. Nakanishi, Y.; Matsugaki, A.; Kawahara, K.; Ninomiya, T.; Sawada, H.; Nakano, T. Unique arrangement of bone matrix orthogonal to osteoblast alignment controlled by Tspan11-mediated focal adhesion assembly. Biomaterials 2019, 209, 103-110. [CrossRef]

20. Wang, P.; Sin, W.J.; Nai, M.L.S.; Wei, J. Effects of processing parameters on surface roughness of additive manufactured Ti-6Al-4V via electron beam melting. Materials 2017, 10, 1121. [CrossRef] 
21. Matsugaki, A.; Aramoto, G.; Ninomiya, T.; Sawada, H.; Hata, S.; Nakano, T. Abnormal arrangement of a collagen/apatite extracellular matrix orthogonal to osteoblast alignment is constructed by a nanoscale periodic surface structure. Biomaterials 2015, 37, 134-143. [CrossRef]

22. Nakano, T.; Fujitani, W.; Ishimoto, T.; Lee, J.W.; Ikeo, N.; Fukuda, H.; Kuramoto, K. Formation of new bone with preferentially oriented biological apatite crystals using a novel cylindrical implant containing anisotropic open pores fabricated by the electron beam melting (EBM) method. ISIJ Int. 2011, 51, 262-268. [CrossRef]

23. Nakano, T.; Kan, T.; Ishimoto, T.; Ohashi, Y.; Fujitani, W.; Umakoshi, Y.; Hattori, T.; Higuchi, Y.; Tane, M.; Nakajima, H. Evaluation of bone quality near metallic implants with and without lotus-type pores for optimal biomaterial design. Mater. Trans. 2006, 2233-2239. [CrossRef]

24. Ikeo, N.; Ishimoto, T.; Serizawa, A.; Nakano, T. Control of mechanical properties of three-dimensional Ti-6Al-4V products fabricated by electron beam melting with unidirectional elongated pores. Metall. Mater. Trans. A. 2014, 45, 4293-4301. [CrossRef]

25. Gibson, L.J.; Ashby, M.F. Cellular Solids—Structure and Properties, 2nd ed.; Cambridge University Press: Cambridge, UK, 1999.

26. Su, C.; Yu, H.; Wang, Z.; Yang, J.; Zeng, X. Controlling the tensile and fatigue properties of selective laser melted Ti-6Al-4V alloy by post treatment. J. Alloys Compd. 2021, 857, 157552. [CrossRef]

27. Alaghmandfard, R.; Chalasani, D.; Odeshi, A.; Mohammadi, M. Activated slip and twin systems in electron beam melted Ti-6Al-4V subjected to elevated and high strain rate dynamic deformations. Mater. Charact. 2021, 172, 110866. [CrossRef]

28. Shao, M.; Vijayan, S.; Nandwana, P.; Jinschek, J.R. The effect of beam scan strategies on microstructural variations in Ti-6Al-4V fabricated by electron beam powder bed fusion. Mater. Des. 2020, 196, 109165. [CrossRef]

29. Al-Bermani, S.; Blackmore, M.; Zhang, W.; Todd, I. The origin of microstructural diversity, texture, and mechanical properties in electron beam melted Ti6Al4V. Metal. Mater. Trans. A 2010, 41, 3422-3434. [CrossRef]

30. Ran, J.; Jiang, F.; Sun, X.; Chen, Z.; Tian, C.; Zhao, H. Microstructure and mechanical properties of Ti-6Al-4V fabricated by electron beam melting. Crystals 2020, 10, 972. [CrossRef]

31. Wu, Y.; Sun, L.; Yang, P.; Fang, J.; Li, W. Energy absorption of additively manufactured functionally bi-graded thickness honeycombs subjected to axial loads. Thin-Walled Struct. 2019, 164, 107810. [CrossRef]

32. Zhang, Q.; Lee, P.D.; Singh, R.; Wu, G.; Lindley, T.C. Micro-CT characterization of structural features and deformation behavior of fly ash/aluminum syntactic foam. Acta Mater. 2009, 57, 3003-3011. [CrossRef]

33. Lam, Q.; Patil, D.; Le, T.; Eppley, T.; Salti, Z.; Goss, D.; Grishin, A.; Bhate, D. An examination of the low strain rate sensitivity of additively manufactured polymer, composite and metallic honeycomb structures. Materials 2019, 12, 3455. [CrossRef]

34. Baranowski, P.; Płatek, P.; Antolak-Dudka, A.; Sarzyński, M.; Kucewicz, M.; Durejko, T.; Małachowski, J.; Janiszewski, J.; Czujko, T. Deformation of honeycomb cellular structures manufactured with Laser Engineered Net Shaping (LENS) technology under quasi-static loading: Experimental testing and simulation. Addit. Manuf. 2019, 25, 307-316. [CrossRef]

35. Boonyongmaneerat, Y. Mechanical properties of partially sintered materials. Mater. Sci. Eng. A 2007, 452-453, 773-780. [CrossRef]

36. Li, X.; Tan, Y.H.; Wang, P.; Su, X.; Jean, H.; Herng, T.S.; Ding, J. Metallic microlattice and epoxy interpenetrating phase composites: Experimental and simulation studies on superior mechanical properties and their mechanisms. Compos. Part A Appl. Sci. Manuf. 2020, 135, 105934. [CrossRef]

37. Ikeo, N.; Ishimoto, T.; Hiramoto, N.; Fukuda, H.; Ogisu, H.; Araki, Y.; Nakano, T. Solid/powder clad Ti-6Al-4V alloy with low Young's modulus and high toughness fabricated by electron beam melting. Mater. Trans. 2015, 56, 755-758.

38. Nakano, T.; Ishimoto, T. Powder-based additive manufacturing for development of tailor-made implants for orthopedic applications. KONA 2015, 32, 75-84. [CrossRef]

39. Tilton, M.; Lewis, G.S.; Wee, H.B.; Armstrong, A.; Hast, M.W.; Manogharan, G. Additive manufacturing of fracture fixation implants: Design, material characterization, biomechanical modeling and experimentation. Addit. Manufact. 2020, $33,101137$. [CrossRef] 